\title{
ON GENERATORS OF CRYSTALLOGRAPHIC GROUPS AND ACTIONS ON FLAT ORBIFOLDS
}

\author{
A. ADEM ${ }^{*}$, K. DEKIMPE ${ }^{\dagger}$, N. PETROSYAN ${ }^{* *}$, AND B. PUTRYCZ $^{\dagger}$ \\ ABstract. We find new bounds on the minimal number of generators \\ of crystallographic groups with $p$-group holonomy. We also show that \\ similar bounds exist on the minimal number of generators of the abelian- \\ izations of arbitrary crystallographic groups. As a consequence, we show \\ that this restricts the rank of elementary abelian $p$-groups that can act \\ effectively on closed connected flat orbifolds.
}

\section{INTRODUCTION}

A closed connected flat $n$-orbifold $M$ is a quotient of $\mathbb{R}^{n}$ by a cocompact action of a discrete subgroup of isometries of $\mathbb{R}^{n}$. A group $\Gamma$ admitting such an action is known as a crystallographic group and it is the orbifold fundamental group of $M$. When $\Gamma$ is torsion-free, it is called a Bieberbach group and it is the fundamental group of the corresponding flat manifold. By the first Bieberbach theorem (see [2]), every $n$-dimensional crystallographic group has a normal subgroup $T$ of translations which is a lattice of $\mathbb{R}^{n}$ and the holonomy group $\Gamma / T$ is finite.

According to a result of Gromov, $\sqrt{2 n \pi} 2^{n-2}$ is an upper bound on the minimal number of generators of the fundamental group of any nonnegatively curved $n$-manifold (see $[10$, p. 21]). In case of flat manifolds, the existing bounds are considerably lower. In [4], Dekimpe and Penninckx proved that every $n$-dimensional crystallographic group with elementary abelian $p$ group holonomy, for any odd prime $p$, is generated by $n$ elements. They also showed that the same bound holds for every $n$-dimensional Bieberbach group with elementary abelian 2-group holonomy.

Date: January 25, 2012.

Key words and phrases. crystallographic group, flat orbifold.

2010 Mathematics Subject Classification. 20H15.

* Partially supported by NSERC.

${ }^{\dagger}$ Supported by Research Fund K.U.Leuven.

${ }^{* *}$ Supported by Research Fund K.U.Leuven and FWO-Flanders Research Fellowship. 
The aim of the present paper is to find attainable bounds on the minimal number of generators of crystallographic groups and their abelianizations and apply this in the study of finite subgroups of symmetries of closed connected flat orbifolds.

We always assume that all actions on orbifolds are by orbifold homeomorphisms. Also, throughout, if not specified, $p$ denotes a prime number, $A$ is an elementary abelian $p$-group and $\beta_{1}$ denotes the first Betti number of the crystallographic group under consideration. Let $a=2$ when $p \leq 19$ and $a=3$ otherwise.

Theorem A. Let $\Gamma$ be an $n$-dimensional crystallographic group such that its holonomy group $G$ is a $p$-group. Then $\Gamma$ can be generated by $a(n-$ $\left.\beta_{1}\right) /(p-1)+\beta_{1}$ elements.

We note that this bound is $2 n-\beta_{1}$ when $p=2$ and it is at most $n$ when $p$ is odd. Hence, Theorem A is a generalization and an improvement of Theorem 4.1 of [4].

For any crystallographic group $\Gamma$, the center $Z(\Gamma)$ is a free abelian group of rank $\beta_{1}$ (see [2]). Moreover, the group $\Gamma / Z(\Gamma)$ is a crystallographic group with trivial center. Therefore, the problem of finding bounds on the minimal number of generators of crystallographic groups may be reduced to finding such bounds for crystallographic groups that have trivial center. For such $n$-dimensional crystallographic groups, the theorem asserts that they are $a n /(p-1)$-generated. In fact, we can show that this bound is attained in arbitrarily large dimensions (see Example 2.4).

Given any, possibly trivial, finite abelian group $F$, we denote $r k(F)=$ $\max \left\{\operatorname{rk}\left(F \otimes \mathbb{Z}_{q}\right) \mid\right.$ prime $\left.q\right\}$. Passing to the abelianizations of crystallographic groups, we obtain:

Theorem B. Let $\Gamma$ be an $n$-dimensional crystallographic group with holonomy group $G$ and $F$ be the torsion subgroup of the abelianization $\Gamma_{a b}$ of $\Gamma$. Let $p$ be a prime such that $r k(F)=\operatorname{rk}\left(F \otimes \mathbb{Z}_{p}\right)$ and $\Gamma^{\prime}$ to be the crystallographic subgroup of $\Gamma$ which is the preimage of a Sylow $p$-subgroup of $G$ under the standard projection of $\Gamma$ onto $G$. Then the minimal number of generators of $\Gamma_{a b}$ is at most $2\left(n-\beta_{1}\left(\Gamma^{\prime}\right)\right) /(p-1)+\beta_{1}\left(\Gamma^{\prime}\right)$.

Let us remark that the tables of abelianizations of crystallographic groups in [13] show that the bounds of Theorem B are sometimes sharp in dimensions 2 and 3 .

Using Theorem B together with the fact that a finite group acts effectively on a closed connected flat $n$-orbifold $M$ if and only if it acts effectively and 
isometrically on an orbifold affinely equivalent to $M$ (see Proposition 4.1), we derive:

Theorem C. Let $A$ be an elementary abelian $p$-group. Suppose $A$ acts effectively on a closed connected flat $n$-orbifold $M$. Then $\operatorname{rk}(A) \leq 2 n$ when $p=2$ and $\operatorname{rk}(A) \leq n$ when $p$ is odd. Moreover, if the holonomy of $M$ is a $p$-group and $p$ is odd, then $\operatorname{rk}(A) \leq 2\left(n-\beta_{1}\right) /(p-1)+\beta_{1}$ where $\beta_{1}$ is the first Betti number of $M$.

\section{Proof of Theorem A}

Let $G$ be a group and $M$ be an integral $G$-lattice. We say that $M$ is irreducible if $M \otimes \mathbb{Q}$ is an irreducible $\mathbb{Q} G$-module.

Lemma 2.1. Let $G$ be a nontrivial $p$-group and assume there exists a faithful irreducible integral $G$-lattice $M$. Then there exists an integer $\alpha \geq 0$ such that $\operatorname{rk}(M)=p^{\alpha}(p-1)$ and every subgroup of $G$ can be generated by $p^{\alpha}$ elements.

Proof. The $G$-module $M \otimes \mathbb{Q}$ is faithful and irreducible. So, by Corollary 1.11 of [5], $\exists \alpha \geq 0$ such that $\operatorname{rk}(M)=p^{\alpha}(p-1)$. Let $n=\operatorname{rk}(M)$. Viewing $G$ as a subgroup of $\mathrm{GL}(n, \mathbb{Q})$, let $P$ be a maximal $p$-subgroup of $\mathrm{GL}(n, \mathbb{Q})$ containing $G$. The representation of $P$ into $G L(n, \mathbb{Q})$ is irreducible. Now, it is a standard result (see $[8, \S 4.5$, p. 93]) that

$$
P \cong \underbrace{C_{p} \imath \cdots \imath C_{p}}_{\alpha+1} .
$$

But, by a result of Hulse (see [7, Theorem A]), any subgroup of such an iterated wreath product can be generated by $p^{\alpha}$ elements.

Proposition 2.2. Let $G$ be a nontrivial $p$-group and $M$ be a faithful integral $G$-lattice of rank $n$. Let $k=\operatorname{rk}\left(M^{G}\right)$. Then every subgroup of $G$ can be generated by $(n-k) /(p-1)$ elements.

Proof. Denote $M_{\mathbb{Q}}=M \otimes \mathbb{Q}$. As a $\mathbb{Q} G$-module, it decomposes into $\left(\oplus_{i=1}^{l} V_{i}\right) \oplus$ $\mathbb{Q}^{k}$ where $n_{1}+\cdots+n_{l}=n-k$ for $n_{i}=\operatorname{dim}\left(V_{i}\right)$, and each $V_{i}$ is an irreducible $\mathbb{Q} G$-module. For each $i$, let $\psi_{i}: G \rightarrow \mathrm{GL}_{n_{i}}(\mathbb{Q})$ be the induced irreducible representation. Since the $\mathbb{Q} G$-module $M_{\mathbb{Q}}$ is faithful, these representations determine a monomorphism:

$$
\begin{aligned}
\psi: G & \hookrightarrow \psi_{1}(G) \times \cdots \times \psi_{l}(G), \\
g & \mapsto\left(\psi_{1}(g), \ldots, \psi_{l}(g)\right) .
\end{aligned}
$$


We claim that every subgroup of the group $D:=\psi_{1}(G) \times \cdots \times \psi_{l}(G)$ can be generated by $(n-k) /(p-1)$ elements. We proceed by induction on $l$. Let $S$ be a subgroup of $D$. By the previous lemma, each subgroup of $\psi_{i}(G)$ can be generated by $n_{i} /(p-1)$ elements. Hence, $l=1$ case is clear. Now, let $l>1$ and consider the standard projection $\pi: \psi_{1}(G) \times \cdots \times \psi_{l}(G) \rightarrow \psi_{l}(G)$. By induction, $\operatorname{ker}\left(\left.\pi\right|_{S}\right)$ is generated by $\left(n_{1}+\cdots+n_{l-1}\right) /(p-1)$ elements and $\pi(S)$ is generated by $n_{l} /(p-1)$ elements. Therefore, $S$ can be generated by $\left(n_{1}+\cdots+n_{l-1}\right) /(p-1)+n_{l} /(p-1)=(n-k) /(p-1)$ elements.

Lemma 2.3. Let $C_{p}$ be a cyclic group of order $p$ and let $M$ be a $\mathbb{Z} C_{p^{-}}$ lattice of dimension $n$ such that $M^{C_{p}}=0$. Then $M$, as a $C_{p^{-}}$-module, can be generated by $(a-1) n /(p-1)$ elements.

Proof. By hypothesis, there exists a family of $C_{p}$-sublattices:

$$
0=V_{0} \subset V_{1} \subset \cdots \subset V_{m}=\mathbb{Z}^{n}
$$

such that each quotient $Q_{i}=V_{i} / V_{i-1}$ is an irreducible, nontrivial $C_{p}$-lattice. It is well-known that every irreducible $\mathbb{Z} C_{p}$-module is of dimension $p-1$ and it is isomorphic to an ideal $I$ in $\mathbb{Z}[\zeta]$ where $\zeta$ is a primitive $p$-th root of unity (see [3]). For $p \leq 19$, the ideal class group of $\mathbb{Z}[\zeta]$ is trivial (see $[14$, 29.1.3]). Therefore, $I$ is a principal ideal and $I \cong \mathbb{Z}[\zeta]$, which is 1 -generated. For $p>19$, since $\mathbb{Z}[\zeta]$ is a Dedekind domain, every ideal is 2-generated (see $[14,7.1-2])$. This shows that each $Q_{i}$ is $(a-1)$-generated. The result now follows by a simple induction on $m$.

Example 2.4. Let $C_{p}$ be a cyclic group of order $p$ and let $I$ be an irreducible $\mathbb{Z} C_{p}$-module. According to the above discussion, as a $C_{p}$-module, $I$ is $(a-1)$ generated. Define $\Gamma$ to be the semidirect product group $I \rtimes C_{p}$. Then $\Gamma$ is $(p-1)$-dimensional crystallographic group. Since $C_{p}$ is 1-generated, $\Gamma$ can be generated by $a$ elements. When $p \leq 19$, this means that $\Gamma$ is 2 -generated and it is clear that this is the minimal number of generators of $\Gamma$. We leave it as an easy exercise to the reader to check that when $p>19$ and $I$ is not a principal ideal, then $\Gamma$ cannot be 2 -generated. Hence, the bound 3 which we obtain for the minimal number of generators of $\Gamma$ is again optimal.

Proposition 2.5. Let $G$ be a $p$-group and $\mathbb{Z}^{n} \longmapsto \Gamma \rightarrow G$ be an extension of groups such that the induced representation $\phi: G \rightarrow \mathrm{GL}_{n}(\mathbb{Z})$ is faithful. Then $\mathbb{Z}^{n}$, as a $G$-module, can be generated by $(a-1)\left(n-\beta_{1}\right) /(p-1)+\beta_{1}$ elements. 
Proof. Recall that $\operatorname{rk}\left(\left(\mathbb{Z}^{n}\right)^{G}\right)=\beta_{1}$. There exists a family of $G$-sublattices:

$$
\left(\mathbb{Z}^{n}\right)^{G}=W_{0} \subset W_{1} \subset \cdots \subset W_{l}=\mathbb{Z}^{n}
$$

such that each quotient $M_{i}=W_{i} / W_{i-1}$ is an irreducible, nontrivial $G$ lattice. Let $C_{p}$ be a central subgroup of the quotient of $G$ by the kernel of the action of $G$ on $M_{i}$. Since $M_{i}^{C_{p}}$ is a $G$-submodule of $M_{i}$, it must be trivial. So, by the previous lemma, we have that, as a $C_{p}$-module and hence also as a $G$-module, each $M_{i}$ is $(a-1) \operatorname{rk}\left(M_{i}\right) /(p-1)$-generated. Again, an easy induction on $l$ finishes the proof.

Proof of Theorem A. Let $\rho: G \rightarrow \mathrm{GL}(T)$ be the holonomy representation of $\Gamma$. Since $\Gamma$ is crystallographic, $\rho$ is faithful. By Proposition 2.2, $G$ can be generated by $\left(n-\beta_{1}\right) /(p-1)$ elements. According to Proposition 2.5, $T$ as a $G$-module, can be generated by $(a-1)\left(n-\beta_{1}\right) /(p-1)+\beta_{1}$. Hence, $\Gamma$ can be generated by $a\left(n-\beta_{1}\right) /(p-1)+\beta_{1}$ elements.

\section{Proof of Theorem B}

Lemma 3.1. Let $G$ be a $p$-group and let $M$ be a $\mathbb{Z} G$-lattice of dimension $n$ such that $M^{G}=0$. Then, $M_{G}$ is an abelian $p$-group of order at most $p^{n /(p-1)}$.

Proof. Let us first assume that $G=C_{p}$. By hypothesis, there exists a family of $C_{p}$-sublattices:

$$
0=V_{0} \subset V_{1} \subset \cdots \subset V_{l}=M
$$

such that each quotient $Q_{i}=V_{i} / V_{i-1}$ is an irreducible, nontrivial $C_{p}$-lattice. Also, $\left(Q_{i}\right)_{C_{p}} \cong \mathbb{Z}_{p}$ (see [2, Ex. IV.4.5]). Proceeding by induction on $l$, we may assume that $\left(V_{l-1}\right)_{C_{p}}$ is a $p$-group of order at most $p^{\left(n-\operatorname{dim}\left(Q_{l}\right)\right) /(p-1)}=$ $p^{(n-(p-1)) /(p-1)}$. Now, by the exact sequence:

$$
\left(V_{l-1}\right)_{C_{p}} \rightarrow M_{C_{p}} \rightarrow\left(Q_{l}\right)_{C_{p}} \rightarrow 0,
$$

we can deduce that $M_{C_{p}}$ is a finite $p$-group of order at most $p \cdot p^{(n-(p-1)) /(p-1)}=$ $p^{n /(p-1)}$. In general, there exists a family of $G$-sublattices:

$$
0=M_{0} \subset M_{1} \subset \cdots \subset M_{l}=M
$$

such that each quotient $N_{i}=M_{i} / M_{i-1}$ is an irreducible, nontrivial $G$-lattice. We take a quotient of $G$ by the kernel of the action of $G$ on $N_{i}$ and let $C_{p}$ be a central subgroup of this quotient. Since $N_{i}$ is irreducible $G$-module, it implies $\left(N_{i}\right)^{C_{p}}=0$. So, by the previous case, $\left(N_{i}\right)_{C_{p}}$ and hence $\left(N_{i}\right)_{G}$, is a $p$-group of order at most $p^{\mathrm{rk}\left(N_{i}\right) /(p-1)}$. The result now follows from a similar induction as before. 
Lemma 3.2. Let $G$ be a $p$-group and let $M$ be an $n$-dimensional $\mathbb{Z} G$ lattice and denote $\operatorname{dim}\left(M^{G}\right)=k$. Then, $\left(M / M^{G}\right)_{G}$ is an abelian $p$-group of order at most $p^{(n-k) /(p-1)}$. Consequently, the rank of $M_{G} \otimes \mathbb{Z}_{p}$ is at most $(n-k) /(p-1)+k$.

Proof. One can easily observe that $\left(M / M^{G}\right)$ is torsion-free and $\left(M / M^{G}\right)^{G}=$ 0 . Hence, by Lemma $3.1,\left(M / M^{G}\right)_{G}$ is an abelian $p$-group of order at most $p^{(n-k) /(p-1)}$. Now, let us consider the exact sequence:

$$
\left(M^{G}\right)_{G} \rightarrow M_{G} \rightarrow\left(M / M^{G}\right)_{G} \rightarrow 0 .
$$

Since $\left(M^{G}\right)_{G} \cong M^{G}$, the rank of $\left(M^{G}\right)_{G} \otimes \mathbb{Z}_{p}$ equals $k$. Also, $\left(M / M^{G}\right)_{G} \otimes \mathbb{Z}_{p}$ has rank at most $(n-k) /(p-1)$. This implies that the rank of $M_{G} \otimes \mathbb{Z}_{p}$ is at most $(n-k) /(p-1)+k$.

Proof of Theorem B. By a standard decomposition of abelian groups:

$$
\Gamma_{a b} \cong \mathbb{Z}^{k} \times \mathbb{Z}_{d_{1}} \times \cdots \times \mathbb{Z}_{d_{s}}
$$

where $k \in \mathbb{Z}_{\geq 0}, d_{i} \in \mathbb{Z}_{\geq 2}$ and $d_{i}$ divides $d_{i+1}$ for each $i$. Note that $\Gamma_{a b}$ is $(s+k)$-generated and the prime $p$ divides each $d_{i}$. By a homological transfer and restriction argument, it follows that there is a monomorphism from $H_{1}\left(\Gamma, \mathbb{Z}_{p}\right)$ into $H_{1}\left(\Gamma^{\prime}, \mathbb{Z}_{p}\right)$. Hence, $s+k=\operatorname{rk}\left(\Gamma_{a b} \otimes \mathbb{Z}_{p}\right) \leq \operatorname{rk}\left(\Gamma_{a b}^{\prime} \otimes \mathbb{Z}_{p}\right)$.

Next, we let $P$ be the holonomy group of $\Gamma^{\prime}$ and note that $T$ is also the subgroup of translations of $\Gamma^{\prime}$. By the 5 -term homological exact sequence associated to the extension $T \longmapsto \Gamma^{\prime} \rightarrow P$, we obtain:

$$
\cdots \rightarrow H_{1}\left(T, \mathbb{Z}_{p}\right)_{P} \rightarrow H_{1}\left(\Gamma^{\prime}, \mathbb{Z}_{p}\right) \rightarrow H_{1}\left(P, \mathbb{Z}_{p}\right) \rightarrow 0
$$

which implies:

$$
\operatorname{rk}\left(\Gamma_{a b}^{\prime} \otimes \mathbb{Z}_{p}\right) \leq \operatorname{rk}\left(P_{a b} \otimes \mathbb{Z}_{p}\right)+\operatorname{rk}\left(T \otimes \mathbb{Z}_{p}\right)_{P} .
$$

By Proposition 2.2 , it follows that $P_{a b} \otimes \mathbb{Z}_{p}$ can be generated by $(n-$ $\left.\beta_{1}\left(\Gamma^{\prime}\right)\right) /(p-1)$ elements. We claim that $\operatorname{rk}\left(T \otimes \mathbb{Z}_{p}\right)_{P} \leq\left(n-\beta_{1}\left(\Gamma^{\prime}\right)\right) /(p-$ 1) $+\beta_{1}\left(\Gamma^{\prime}\right)$.

To this end, consider the short exact sequence:

$$
0 \rightarrow T \stackrel{p}{\rightarrow} T \rightarrow T \otimes \mathbb{Z}_{p} \rightarrow 0
$$

Applying the coinvariants functor $(-)_{P}$, we obtain the exact sequence:

$$
T_{P} \stackrel{p}{\rightarrow} T_{P} \rightarrow\left(T \otimes \mathbb{Z}_{p}\right)_{P} \rightarrow 0 .
$$

This shows that $\left(T \otimes \mathbb{Z}_{p}\right)_{P} \cong T_{P} \otimes \mathbb{Z}_{p}$. But, by the previous lemma, $T_{P} \otimes \mathbb{Z}_{p}$ has rank at most $\left(n-\beta_{1}\left(\Gamma^{\prime}\right)\right) /(p-1)+\beta_{1}\left(\Gamma^{\prime}\right)$, which finishes the claim. 
Combining with a previous inequality, we obtain $s+k \leq \operatorname{rk}\left(\Gamma_{a b}^{\prime} \otimes \mathbb{Z}_{p}\right) \leq$ $2\left(n-\beta_{1}\left(\Gamma^{\prime}\right)\right) /(p-1)+\beta_{1}\left(\Gamma^{\prime}\right)$.

\section{Proof of Theorem C}

First, we need a similar result as Theorem 6 of [9] which applies to orbifolds.

Proposition 4.1. Let $F$ be a finite group. Suppose it acts effectively on a closed connected flat $n$-orbifold $M$. Then $F$ can also act effectively and isometrically on an orbifold affinely equivalent to $M$.

Proof. The orbifold $M$ arises as a quotient of $\mathbb{R}^{n}$ by a discrete group $\Gamma$ acting effectively, isometrically, properly discontinuously and cocompactly on $\mathbb{R}^{n}$ (see $[12,13.3 .3-4]$ ). Then $\Gamma$ is an $n$-dimensional crystallographic group and the natural quotient map $p: \mathbb{R}^{n} \rightarrow \mathbb{R}^{n} / \Gamma$ is an orbifold covering map where $\mathbb{R}^{n}$ is the orbifold universal cover.

On the other hand, the quotient $M / F$ is a closed connected orbifold and the quotient map $q: M \rightarrow M / F$ is an orbifold covering map (see [11, p. 40, Ex. 2.18], [15, 13.2]). So, the projection $q \circ p: \mathbb{R}^{n} \rightarrow M / F$ is again an orbifold covering map (see $[15,13.2 .4])$. This shows that there exists an extension $\Gamma \longmapsto \bar{\Gamma} \rightarrow F$ where $\bar{\Gamma}$ is the orbifold fundamental group of $M / F$.

We claim that $\bar{\Gamma}$ is an $n$-dimensional crystallographic group. According to theorems of Bieberbach ([2]) and Zassenhaus ([16]), a finitely generated virtually abelian group is crystallographic if and only if it does not contain a finite nontrivial normal subgroup. Let us suppose that $S$ is a finite normal subgroup of $\bar{\Gamma}$. Let $T$ be the subgroup of translations of $\Gamma$ and $\Gamma^{\prime}:=T S \leq \bar{\Gamma}$. Since $T \cap S=1$, there is an extension $T \longmapsto \Gamma^{\prime} \rightarrow S$. This extension induces an orbifold covering map $\mathbb{R}^{n} / T \rightarrow \mathbb{R}^{n} / \Gamma^{\prime}$ which arises from an action of $S$ on the $n$-torus $\mathbb{R}^{n} / T$. Let $K$ be the kernel of this action. By passing to a subgroup of $S$, we can without loss of generality assume that $S=$ $K$. Then $\mathbb{R}^{n} / \Gamma^{\prime}$ is equivalent as an orbifold to $\mathbb{R}^{n} / T$ and hence they have isomorphic orbifold fundamental groups. This shows that $\Gamma^{\prime} \cong T$ and so $K=1$. We deduce that $S$ acts effectively on $\mathbb{R}^{n} / T$. Then, by a result of Lee and Raymond (see [9, Cor., p. 256]) the extension $T \longmapsto \Gamma^{\prime} \rightarrow S$ is admissible and therefore $\Gamma^{\prime}$ is a crystallographic group (see [9, Prop. 2]). Since $S$ is a finite normal subgroup of $\Gamma^{\prime}, S$ must be trivial. We can therefore conclude that $\bar{\Gamma}$ is an $n$-dimensional crystallographic group.

Let $\bar{\Gamma}$ act cocompactly, effectively, isometrically and properly discontinuously on $\mathbb{R}^{n}$ and denote by $\mathbb{R}^{n} / / \Gamma$ the quotient of the action of the subgroup 
$\Gamma$ of $\bar{\Gamma}$ on $\mathbb{R}^{n}$. We observe that the extension $\Gamma \longmapsto \bar{\Gamma} \rightarrow F$ gives rise to an effective and isometric action of $F$ on the orbifold $\mathbb{R}^{n} / / \Gamma$. By the second Bieberbach theorem (see [2]), $\mathbb{R}^{n} / \Gamma$ and $\mathbb{R}^{n} / / \Gamma$ are affinely equivalent. This finishes the proof.

Proof of Theorem C. By the proposition, we can assume that $A$ acts isometrically on $M$. Let $\bar{M}=M / A$. It is again a compact flat orbifold. Let $\bar{\Gamma}=\pi_{1}^{\text {orb }}(\bar{M})$. Then $\bar{M} \cong \mathbb{R}^{n} / \bar{\Gamma}$ and $\bar{\Gamma}$ is again a crystallographic group. We also have an extension $\Gamma \longmapsto \bar{\Gamma} \rightarrow A$. Observe that the epimorphism of $\bar{\Gamma}$ onto $A$ factors through the group $H:=\bar{\Gamma} / T$ where $T$ is the subgroup of translations of $\Gamma$. Let $\phi: H \rightarrow A$ be the induced epimorphism and note that the restriction of $\phi$ to a Sylow $p$-subgroup $P$ of $H$ is again surjective. Let $\Gamma^{\prime}$ be the preimage of $P$ under the projection of $\bar{\Gamma}$ onto $H$. Then $\Gamma^{\prime}$ is an $n$-dimensional crystallographic group with a $p$-group holonomy. So, by applying Theorem B to $\Gamma^{\prime}$ it follows that $\Gamma_{a b}^{\prime}$ is $n$-generated when $p$ is odd and $2 n$-generated when $p=2$. But since $\Gamma_{a b}^{\prime}$ surjects onto $A$, this gives us the desired bounds on $\operatorname{rk}(A)$.

If $\Gamma$ has only $p$-group holonomy and $p$ is odd, then $P=H$ and $\Gamma^{\prime}=\bar{\Gamma}$. In this case, Theorem B entails $\operatorname{rk}(A) \leq 2\left(n-\bar{\beta}_{1}\right) /(p-1)+\bar{\beta}_{1} \leq 2\left(n-\beta_{1}\right) /(p-$ 1) $+\beta_{1}$ where the second inequality follows from the fact that $\beta_{1} \geq \bar{\beta}_{1}$.

\section{REFERENCES}

[1] Bieberbach, L., Über die Bewegungsgruppen der Euklidischen Raume I, Math. Ann. (1911), 70, 297-336.

[2] Charlap, L., Bieberbach Groups and Flat Manifolds, Universitext, SpringerVerlag, Berlin, (1986).

[3] Curtis, C.W., and Reiner, I., Representation Theory of Finite Groups and Associative Algebras, Wiley-Interscience (1987).

[4] Dekimpe, K., and Penninckx, P., On the number of generators of a Bieberbach group, Communications in Algebra 37, no. 12, (2009), 4332-4345.

[5] Eckmann, B., and Mislin, G., Rational representations of finite groups and their Euler class, Math. Ann. 245 (1979), 45-54.

[6] Hiss, G., and Szczepanśki, A., Flat manifolds with only finitely many affinities, Bull. Polish Acad. Sci. Math. 45 (1997), no. 4, 349-357.

[7] Hulse, J.A., Generators for subgroups of wreath products, Proc. Edinburgh Math. Soc. 22 (1979), 195-199.

[8] Leedham-Green, C.R., and McKay, S., The Structure of Groups of Prime Power Order, Oxford Univ. Press, Oxford, (2002).

[9] Lee, K.B., and Raymond, F., Topological, affine and isometric actions on flat Riemannian manifolds, J. Differential Geom. 16 (1981), 255-269.

[10] Meyer, W.T., Toponogov's Theorem and Applications, Lecture Notes, College on Differential Geometry, Trieste (1989). 
[11] Moerdijk, I., and Mrčun, J., Introduction to foliations and Lie groupoids Cambridge Studies in Advanced Mathematics, 91. Cambridge University Press, Cambridge, (2003).

[12] Ratcliffe, J.G., Foundations of hyperbolic manifolds, 2nd edition, Graduate Text in Math., 149, Springer-Verlag, Berlin, Heidelberg, and New York (2006).

[13] Ratcliffe, J.G., and Tschantz, S.T., Abelianization of space groups, Acta Cryst. (2009). A65, 18-27.

[14] Ribenboim, P., Classical theory of algebraic numbers, Springer-Verlag, Heidelberg (2001).

[15] Thurston, W., The geometry and topology of three-manifolds, Princeton University lecture notes (1978-1981).

[16] Zassenhaus, H. Über einen Algorithmus zur Bestimmung der Raumgruppen, Commentarii Mathematici Helvetici 21 (1948) 117-141.

Department of Mathematics, University of British Columbia, Vancouver, CANADA

E-mail address: adem@math.ubc.ca

Department of Mathematics, Katholieke Universiteit Leuven, Kortrijk, BELGIUM

E-mail address: Karel.Dekimpe@kuleuven-kortrijk.be

E-mail address: Nansen.Petrosyan@kuleuven-kortrijk.be

Department of Mathematics, Katholieke Universiteit Leuven, Kortrijk, BELGiUM

Institute of Mathematics, University of Gdańsk, Gdańsk, Poland

E-mail address: Bartosz.Putrycz@mat.ug.edu.pl 